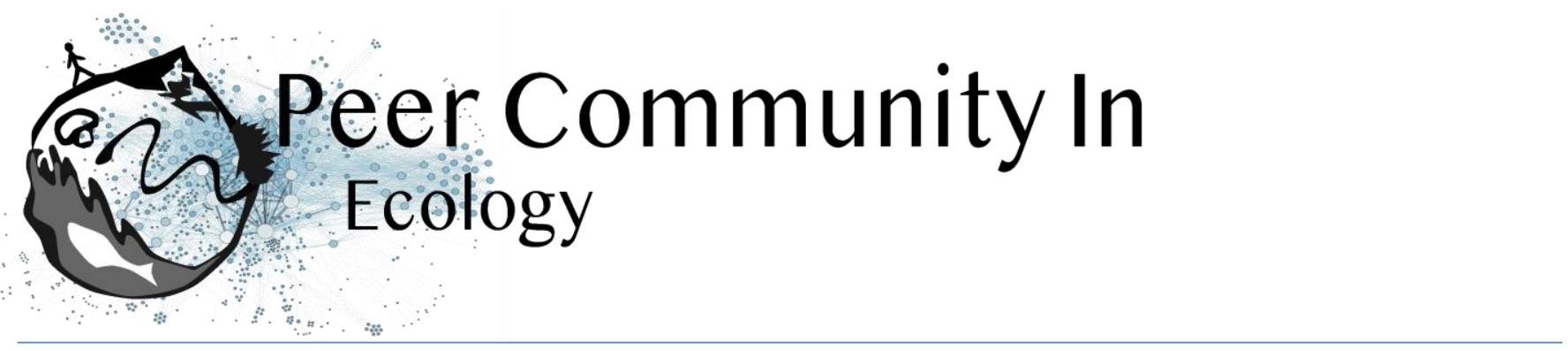

\title{
Doomed by your partner: when mutualistic interactions are like an evolutionary millstone around a species' neck
}

\author{
Sylvain Billiard based on reviews by 2 anonymous reviewers \\ A recommendation of:
}

Plant eco-evolution weakens mutualistic interaction with declining pollinator populations

Avril Weinbach, Nicolas Loeuille, Rudolf P. Rohr (2021), bioRxiv, 570580, ver. 5 peerreviewed and recommended by Peer Community in Ecology

https://doi.org/10.1101/570580

\section{Open Access}

Submitted: 05 September 2019, Recommended: 04 October 2021

Cite this recommendation as:

Sylvain Billiard (2021) Doomed by your partner: when mutualistic interactions are like an evolutionary millstone around a species' neck. Peer Community in Ecology, 100089. https://doi.org/10.24072/pci.ecology.100089

\section{Published: 20 October 2021}

Copyright: This work is licensed under the Creative Commons Attribution-NoDerivatives 4.0 International License. To view a copy of this license, visit http://creativecommons.org/licen ses/by-nd/4.0/

\section{Recommendation}

Mutualistic interactions are the weird uncles of population and community ecology. They are everywhere, from the microbes aiding digestion in animals' guts to animal-pollination services in ecosystems; They increase productivity through facilitation; They fascinate us when small birds pick the teeth of a big-mouthed crocodile. Yet, mutualistic interactions are far less studied and understood than competition or predation. Possibly because we are naively convinced that there is no mystery here: isn't it obvious that mutualistic interactions necessarily facilitate species coexistence? Since mutualistic species benefit from one another, if one species evolves, the other should just follow, isn't that so?

It is not as simple as that, for several reasons. First, because simple mutualistic LotkaVolterra models showed that most of the time mutualistic systems should drift to infinity and be unstable (e.g. Goh 1979). This is not what happens in natural populations, so something is missing in simple models. At a larger scale, that of communities, this is even worse, since we are still far from understanding the link between the topology of mutualistic networks and the stability of a community. Second, interactions are contextdependent: mutualistic species exchange resources, and thus from the point of view of one species the interaction is either beneficial or not, depending on the net gain of energy (e.g. Holland and DeAngelis 2010). In other words, considering interactions as mutualistic per se is too caricatural. Third, since evolution is blind, the evolutionary response of a species to an environmental change can have any effect on its mutualistic partner, and not necessarily a neutral or positive effect. This latter reason is particularly highlighted by the paper by A. Weinbach et al. (2021).

Weinbach et al. considered a simple two-species mutualistic Lotka-Volterra model and analyzed the evolutionary dynamics of a trait controlling for the rate of interaction between the two species by using the classical Adaptive Dynamics framework. They 


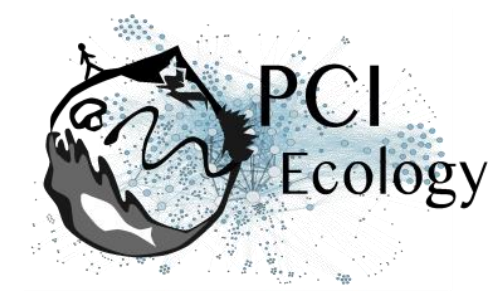

showed that, depending on the form of the trade-off between this interaction trait and its effect on the intrinsic growth rate, several situations can occur at evolutionary equilibrium: species can stably coexist and maintain their interaction, or the interaction traits can evolve to zero where species can coexist without any interactions.

Weinbach et al. then investigated the fate of the two-species system if a partner species is strongly affected by environmental change, for instance, a large decrease of its growth rate. Because of the supposed trade-off between the interaction trait and the growth rate, the interaction trait in the focal species tends to decrease as an evolutionary response to the decline of the partner species. If environmental change is too large, the interaction trait can evolve to zero and can lead the partner species to extinction. An "evolutionary murder".

Even though Weinbach et al. interpreted the results of their model through the lens of plant-pollinators systems, their model is not specific to this case. On the contrary, it is very general, which has advantages and caveats. By its generality, the model is informative because it is a proof of concept that the evolution of mutualistic interactions can have unexpected effects on any category of mutualistic systems. Yet, since the model lacks many specificities of plant-pollinator interactions, it is hard to evaluate how their result would apply to plant-pollinators communities.

I wanted to recommend this paper as a reminder that it is certainly worth studying the evolution of mutualistic interactions, because i) some unexpected phenomenons can occur, ii) we are certainly too naive about the evolution and ecology of mutualistic interactions, and iii) one can wonder to what extent we will be able to explain the stability of mutualistic communities without accounting for the co-evolutionary dynamics of mutualistic species.

\section{References}

Goh BS (1979) Stability in Models of Mutualism. The American Naturalist, 113, 261275. http://www.jstor.org/stable/2460204.

Holland JN, DeAngelis DL (2010) A consumer-resource approach to the density-dependent population dynamics of mutualism. Ecology, 91, 1286-1295. https://doi.org/10.1890/09-1163.1

Weinbach A, Loeuille N, Rohr RP (2021) Eco-evolutionary dynamics further weakens mutualistic interaction and coexistence under population decline. bioRxiv, 570580, ver. 5 peer-reviewed and recommended by Peer Community in Ecology. https://doi.org/10.1101/570580

\section{Reviews}

Toggle reviews

\section{Evaluation round \#2}

15 Jul 2021

DOI or URL of the preprint: https://doi.org/10.1101/570580

Version of the preprint: 3

\section{Author's Reply}




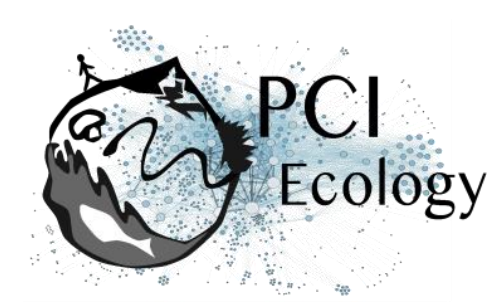

\section{Decision by Sylvain Billiard}

Dear authors,

I will be happy to recommend your manuscript pending minor revisions (see the attached file for details).

Best regards,

Sylvain Billiard

Download recommender's annotations

Reviewed by anonymous reviewer, 05 Jul 2021

Download the review

\section{Evaluation round \#1}

03 Nov 2019

DOI or URL of the preprint: https://doi.org/10.1101/570580

Version of the preprint: 2

\section{Author's Reply}

Download author's reply

\section{Decision by Sylvain Billiard}

Dear authors, Firstly, I wish to apologize for the too long duration of this review process due to the difficulty in finding two appropriate and qualified reviewers. Two reviewers and myself have thoroughly read your paper. We all find that your paper has the potential to be recommended by PCI Ecology. However, we all find that it lacks much clarity in the concepts used and the definitions, and in the model assumptions and calculations. You will find in the attached reviews, technical and conceptual comments and corrections, as well as other aspects such as suggestion of improvement of the paper organization. It is important to address these questions and comments in order to satisfy a large audience from empiricists to theoreticians. You will also find detailed comments from my own reading of your paper. You will see that I insist on the following aspect: I think that your model is very general and, as a consequence, lacks specificity regarding the core of your message: the evolution of plant pollinator interactions. I would suggest to be less specific and more general in the introduction (it is not necessary to invoke global changes, pollinator declines, etc. to justify your work) and talk about what you really do: 1) develop an adaptive dynamics model for the evolution of the interaction rate between two mutualistic species, and especially study their conditions for coexistence, and 2) apply it to a specific question inspired from plant-pollinator interactions in a context of environmental degradation. I find particularly important to compare your model to previous ones which can be very close, for instance Ferrière et al. 2002 (Proceedings of the Royal Society B). I would be happy to reconsider a revised version of your paper for a potential recommendation in $\mathrm{PCl}$ Ecology. Best regards, Dr. Sylvain Billiard

**Additional requirements of the managing board**: As indicated in the 'How does it work?' section and in the code of conduct, please make sure that: -Data are available to readers, either in the text or through an open data repository such as Zenodo (free), Dryad or some other institutional repository. Data must be reusable, thus metadata or accompanying text must carefully describe the data. -Details on quantitative 


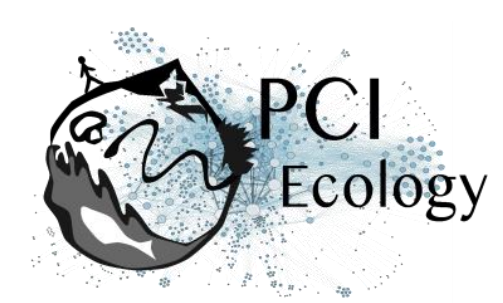

analyses (e.g., data treatment and statistical scripts in R, bioinformatic pipeline scripts, etc.) and details concerning simulations (scripts, codes) are available to readers in the text, as appendices, or through an open data repository, such as Zenodo, Dryad or some other institutional repository. The scripts or codes must be carefully described so that they can be reused. -Details on experimental procedures are available to readers in the text or as appendices. -Authors have no financial conflict of interest relating to the article. The article must contain a "Conflict of interest disclosure" paragraph before the reference section containing this sentence: "The authors of this preprint declare that they have no financial conflict of interest with the content of this article." If appropriate, this disclosure may be completed by a sentence indicating that some of the authors are $\mathrm{PCI}$ recommenders: "XXX is one of the $\mathrm{PCI} X X X$ recommenders."

Download recommender's annotations

Reviewed by anonymous reviewer, 07 Oct 2019

Download the review

Reviewed by anonymous reviewer, 31 Oct 2019

Download the review 